\title{
Intervenção em Turmas de Jovens e Adultos numa Escola da Rede Pública do Recife, Multidisciplinaridade e Uso da Ferramenta Blockly Games - Um Relato de Experiência.
}

\author{
Marcela Portela S. de Figueiredo1, Elidiene Gomes de Oliveira Lima2, Gilberto \\ Amado de Azevedo Cysneiros Filho3
}

1Programa de Pós-graduação em Biometria e Estatística Aplicada - Universidade Federal Rural de Pernambuco (UFRPE)- Recife - PE - Brazil

2Escola Municipal Senador José Ermírio de Moraes

3Departamento de Estatística e InformáticaUniversidade Federal Rural de Pernambuco (UFRPE) - Recife, PE - Brazil portela.marcela.producao@gmail.com, elidienegomes@hotmail.com, g.cysneiros@gmail.com

\begin{abstract}
Digital games are increasingly applied as teaching tool support. How to use digital games in the educational environment is still a research area. This paper describes an experience of development of a game adapted of Blockly Games and applied with students of classes of Education of Young and Adults. The goal of the game is to teach subjects related to Mathematics, Environment Educational and Programming. The result of the experiment was that 43,75\% of the students were satisfied or very satisfied with the game. In relation to learning perception, $81,25 \%$ believe that they learned mathematics, 87,5\% believe that they learned logic and 93\% believe they learned recycling notions.

Resumo. Jogos digitais são cada vez mais aplicados como ferramentas de suporte ao ensino. Como aplicar jogos digitais no ambiente educacional ainda é uma área de pesquisa. Esse artigo descreve uma experiência de desenvolvimento de um jogo adaptado do Blockly Games e aplicá-lo com alunos de turmas de Educação de Jovens e Adultos (EJA). O objetivo do jogo é ensinar assuntos relacionados a Matemática, Educação Ambiental e Programação. $O$ resultado do experimento foi que $43,75 \%$ dos estudantes se mostraram satisfeitos ou muito satisfeitos com o jogo. Em relação à percepção de aprendizado, 81,25\% acreditam terem aprendido matemática, 87,5\% acreditam terem aprendido lógica e $93 \%$ acreditam ter aprendido noções de reciclagem.
\end{abstract}

\section{Introdução}

A gamificação pode ser definida, segundo Cavadas et al. (2017) como a aprendizagem que utiliza a mecânica de design de jogos. Seu uso tem demonstrado indícios que sua utilização no ambiente educacional promove o processo de ensino, gerando motivação e prendendo a atenção dos estudantes em sala de aula [Souza Junior et al. 2018]. Segundo González, Gómez e Echeverri (2017) os elementos de jogos aumentam o espírito criativo do educando, sua motivação, concentração, esforço, lealdade e incentivam o trabalho em equipe. A ludicidade assim, seria uma maneira de motivar o aluno no contexto da 
educação virtual [GONZÁLEZ, GÓMEZ E ECHEVERRI 2017]. A utilização da programação de blocos, utilizando elementos de jogos poderia, desta maneira, motivar o ensino de programação entre jovens e adultos. Estudos vem sendo realizados nesta linha com crianças e adolescentes [GLUSHKOVAL 2016], porém existe uma literatura escassa em relação ao estudo do ensino de Jovens e Adultos e a Gamificação, em particular programação em blocos, no Brasil.

O Blockly é uma programação visual, classificada como programação em blocos. Ela é uma biblioteca JavaScript, um projeto aberto da Google. Seu uso na educação tem demonstrado que utilizando-se os blocos, os erros de sintaxe da linguagem de programação tradicional são evitados e permitem que o aluno esteja mais focado na implementação dos algoritmos e cenários, deixando um maior espaço para 0 desenvolvimento do pensamento computacional. Ele também possibilita a inserção de alunos de diferentes níveis de conhecimento e experiência em programação [GLUSHKOVAL 2016], sendo assim mais adequado para o uso na Educação de Jovens e Adultos. Existem outras programações em bloco disponíveis no mercado, tais como: Scratch, Snap, Stencyl, MIT App Inventor, Alice, porém o Blockly é descrito na literatura como o mais intuitivo para empilhar os blocos [FRASER 2015], utiliza pop-ups que não podem ser fechados para monitorar as ações do aluno e prescrever ações a serem realizadas [FRASER 2015], o que o torna uma ferramenta melhor para o ensino de programação. Assim, as vantagens em utilizar a programação em blocos para o ensino de programação são: estilo de programação fácil sem erros de sintaxe, resultado rápido, trabalho em linguagem natural, blocos coloridos diferentes, modo de arrastar e soltar possível, muito útil para tablets, ambientes de programação diferentes adequados para alunos de todas as idades e níveis de treinamento e permite o uso de todos os algoritmos básicos em programação [GLUSHKOVAL 2016].

O Blockly Games é uma série de jogos educativos que ensinam programação. Ele foi projetado para pessoas que não tiveram experiência anterior com programação, é especificamente projetado para ser autodidata, ou seja, nenhum professor ou plano de aula é necessário para a dinâmica educacional [FRASER 2015]. No final da sequência de jogos: Puzzle, Maze, Bird, Turtle, entre outros, os jogadores estão prontos para usar linguagens convencionais baseadas em texto [GOOGLE 2019]. O presente trabalho se utilizou do jogo Bird, do Blockly Games, modificando-o para a nova proposta temática do projeto que seria o ensino de matemática, educação ambiental (reciclagem), lógica e programação que foram aplicados ao ensino de Jovens e Adultos, de forma a incutir valores que promovam a manutenção de cidades limpas no estado de PE, além de familiarizar o educando com ferramentas e linguagens de programação, ensinar matemática de maneira lúdica de forma a fazer com que aprendê-la seja prazeroso. No contexto da educação de jovens e adultos essa temática se torna importante pois se tratam de estudantes que podem possuir dificuldades de aprendizagem [MEC 2006], problemas de permanência escolar [MEC 2006], idade destoante do ambiente escolar [MEC 2006] o que faz com que o educando se sinta envergonhado [MEC 2006], ou com dificuldades de aprendizagem por trabalhar bastante [MEC 2006], ou por ter filhos [MEC 2006], além das diferenças de gerações e de integrações tecnológicas e de técnicas de aprendizagem, advinda dessa diferença de idade [MEC 2006], o que faz com que trabalhar com turmas de jovens e adultos seja uma tarefa desafiadora tanto para o professor, quanto para a direção da escola e da família do educando. Vale salientar da necessidade da produção de literatura que aborde a educação de jovens e adultos no Brasil, "não são muitos os 
subsídios escritos destinados a responder às necessidades pedagógicas dos educadores que atuam nas salas de aula da educação de jovens e adultos" [MEC, 2006], justificando sua escolha como público alvo.

\subsection{Trabalhos Relacionados}

Wildner, Franzen e Gomes (2018) desenvolveram uma ferramenta web chamada HelpBlock com o objetivo de ensinar algoritmos em turmas de graduação de faculdades de computação. O HelpBlock utiliza a biblioteca Blockly. Essa ferramenta obteve altos índices de satisfação pelos discentes além de promover a motivação dos educandos, porém o público-alvo se trata de estudantes de graduação e não da turma de Jovens e Adutos brasileiros, público-alvo do presente artigo. Já Leitão e Castro (2018) utilizaram o Scratch em uma oficina, onde os participantes (estudantes dos cursos de Pedagogia e de Matemática, professores da Educação Básica e alunos da Pós-Graduação) desenvolveram jogos com o intuito de ensinar matemática, tais como o "Jogo da Multiplicação" e "Pokémon e as operações" (trata da soma, subtração, multiplicação e divisão), ambos utilizado programação em blocos [LEITÃO e CASTRO 2018]. Hein et al. (2015) exploraram a temática da robótica e desenvolveram uma camada de interface implementada com a biblioteca Blockly, que por sua vez passa informações para o microcontrolador Raspberry Pi que controla o modelo robótico. Embora utilize a temática multidisciplinar para o ensino de Geografia e reciclagem de lixo eletrônio através da programação em blocos, o público-alvo do estudo foi diferente, se tratando de estudantes de terceira e quarta série em escolas de Faro, Portugal.

Silva, Sales e Castro (2018) estudaram o efeito da gamificação com auxílio do ambiente Moodle Help Class Online na aprendizagem de física por estudantes do segundo ano do Ensino Médio dos cursos de Técnico Integrado do Instituto Federal de Educação, Ciência e Tecnologia do Ceará (IFCE). Os autores concluíram que a gamificação promoveu a motivação nos estudantes e a melhora na aprendizagem [Silva, Sales e Castro 2018]. Matos e Matos (2017) relataram que a gamificação proporcionou maior interesse dos estudantes na matéria de Português em turmas do curso profissionalizante de Técnico em Informática do IF Goiano, nas turmas de segundo e terceiro anos. Freitas, Maio e Basílio (2018) construíram uma ferramenta de ensino de matemática chamada ProGame direcionada aos alunos do primeiro ano do ensino médio, ou seja, não foi aplicado em turmas de Jovens e Adultos. Como resultados encontraram que os alunos se sentiram à vontade com os recursos e que pretendem utilizar o ambiente gamificado com frequência [Freitas, Maio e Basílio 2018].

Paganini e Bolzan (2016) elaboraram um jogo de ensino de física voltado para a Educação de Jovens e Adultos, público escolhido pelo presente trabalho. A ferramenta desenvolvida pelos autores se mostrou bastante promissora, porém não utiliza a programação em blocos. Já Oliveira, Bitencourt e Monteiro (2016) dizem utilizar a gamificação em turma de jovens e adultos, porém em um contexto diferente, pois se trata do ensino médio integrado ao nível técnico, além de não utilizar a programação em blocos. A proposta deste trabalho, é a utilização de uma ferramenta para ensino multidisciplinar (matemática, lógica, programação e educação ambiental) com o uso da programação em blocos de forma gamificada e sua aplicação na sala de aula para identificar indícios da sua eficácia na aprendizagem e motivação na turma de Jovens e Adultos - EJA, a qual será melhor explicada na secção de metodologia. 


\section{Metodologia}

Em um primeiro momento foi criada uma página personalizada para o projeto, com o objetivo de apresentar o projeto, e hospedar o jogo, personalizado para o Colégio. A página foi desenvolvida em HTML e CSS. Em seguida foi realizada a modificação do jogo Bird, do Blockly Games personalizando-o para a aplicação no colégio e modificando a temática do jogo, o personagem principal do jogo e seu objetivo final. O jogo é dividido em 10 fases em que cada uma requer um conhecimento mais avançado de matemática e programação, além de desenvolver a consciência ambiental. O jogo é codificado em JavaScript e utiliza o Closure compile da Google. Após essa etapa, foi realizada a instrução de uso do jogo para a professora, que consistiu em um papel de ambientar a professora do colégio com a proposta do projeto e do jogo, assim como utilizá-lo e combinar ideias da maneira de se utilizar essa ferramenta em sala de aula. Após isso, foi aplicado o jogo em sala de aula. A professora efetiva do colégio ficou responsável por administrar a sala de aula, retirando qualquer viés na pesquisa que poderia trazer se um outro profissional fosse responsável por ministrar a aula para turma. Antes de cada fase do jogo foi apresentado o conteúdo necessário para sua execução (ex.: círculo trigonométrico e plano cartesiano). Por último, aplicação de questionário de satisfação do aluno e do professor.

\section{Análise dos Resultados}

A turma do EJA módulo 5 foi acompanhada pela professora que utilizou o data-show para ensinar como jogá-lo, enquanto um aluno ficou em um computador programando em blocos de acordo com a direção (ângulo) que os colegas da turma diziam para ele jogar, ou seja, foi feita uma atividade coletiva em que todos os alunos davam suas opiniões e discutiam entre eles e viam no data-show o resultado das escolhas. Segundo a professora, os estudantes ficaram animados por verem os acertos que realizavam com relação ao conteúdo de matemática no jogo realizado coletivamente. Eles chegaram ao nível 4. Já na turma módulo 4, que teve aula depois desse dia, foi possível a utilização dos tablets com o modem pessoal, sendo a turma dividida em grupos de 3 a 4 pessoas por tablet, para resolver o jogo, porém a maior parte dos grupos não passou da primeira fase devido a velocidade da internet wi-fi que estava lenta devido a problemas na ocasião. No fim da aula, quando a maioria dos estudantes haviam se retirado, um grupo continuou o jogo e os estudantes dessa turma conseguiram chegar até o nível 4, pois a velocidade da internet melhorou porque poucas pessoas estavam usando a internet. Em seguida veio a época das avaliações de fim de ano, e não se pode mais repetir a aplicação do jogo, embora os estudantes o queiram e a professora também. Vale salientar que antes que o jogo fosse introduzido, foram realizadas aulas sobre o conteúdo matemático necessário para a compreensão dos estudantes para cada fase do jogo. A professora também fez uma atividade com o jogo impresso no papel nas primeiras fases. A familiarização realizada pela pesquisadora sobre o jogo Preservando a Natureza para a professora se mostrou fundamental para a apropriação da ferramenta computacional pela mesma, influenciando o planejamento de aula da professora. 


\subsection{Análise descritiva dos dados}

O estudo foi composto por 2 turmas de Jovens e Adultos, módulo 4 e módulo 5, em um universo de 53 estudantes, 16 estudantes foram selecionados por conveniência, por comparecerem ao estabelecimento de ensino nos dias do experimento. 30,19\% dos estudantes, participaram do experimento de uma maneira global (turma 4 e 5 conjuntamente). Destes 53 alunos, 60,38\% dos estudantes são mulheres e os estudantes estão distribuídos em uma faixa de idade de 16 a 76 anos. 35\% dos estudantes estão na faixa etária de 16 a 26 anos. A média de idade foi de 34 anos, com desvio padrão de 15 anos. Em relação à amostra, 62,50\% dos estudantes são mulheres. Ela está distribuída em uma faixa de 17 a 67 anos de idade, onde $75,00 \%$ dos estudantes se encontram numa faixa etária entre 17 e 46 anos, e uma média de 36 anos e desvio padrão de 14 anos aproximadamente.

\section{Discussão}

$100 \%$ dos estudantes da amostra selecionada responderam ao questionário de satisfação. $43,75 \%$ dos estudantes se mostraram satisfeitos ou muito satisfeitos com o jogo Preservando à Natureza, enquanto que 50\% se mostraram indiferentes. Em relação à percepção de aprendizado, 81,25\% dos estudantes acreditam ter aprendido matemática através do jogo, $87,5 \%$ creem terem aprendido lógica, 93\% dos estudantes acreditam ter aprendido noções de meio ambiente (reciclagem), porém $75 \%$ dos estudantes não creem ter aprendido programação. Provavelmente a velocidade da internet pode ter influenciado os resultados, pelo fato dos estudantes terem dificuldade de passar de fase, e assim treinarem pouco a programação em blocos em pequenos grupos, porém se deixa em aberto para um próximo estudo mais aprofundado. $75,00 \%$ dos estudantes chegaram ao nível 4, porém o restante não passou do nível 1, devido à internet. $81,25 \%$ acharam o jogo difícil, porém $100 \%$ dos estudantes gostariam de repetir a experiência com jogos.

Também foram incluídas perguntas sobre a familiarização dos educandos com relação aos aparatos tecnológicos que poderiam ser utilizados na sala de aula para jogar Preservando a Natureza. Apenas 50\% dos estudantes dizem saber utilizar o computador, enquanto que $31,25 \%$ dos discentes sabem utilizar tablet e 56,25\% sabem utilizar o smartphone, o que pode ser uma barreira para o aprendizado utilizando novas tecnologias, e mostra indícios que os estudantes do EJA ainda precisam se familiarizar com tais tecnologias. Os dados também evidenciaram que os estudantes que possuíam os dispositivos em casa constituem em sua maior parte dos estudantes que sabem utilizar os respectivos aparelhos, o que mostra indícios que os estudantes precisam de maior acessibilidade a tablets, computadores e smartphones, em suas próprias residências, para uma maior facilidade de uso em sala de aula.

Obteve-se como resposta do questionário de satisfação do professor, que a docente está satisfeita em relação ao jogo Preservando a Natureza. Segundo a mesma, os estudantes estiveram motivados durante a utilização do jogo. Com relação ao ensino de programação proposto pelo jogo, ela se descreveu satisfeita, e acrescentou que o jogo necessita de um tutorial falando sobre a programação em blocos e como utilizá-la, o significado da linguagem e dos símbolos. Em relação às maiores dificuldades encontradas 
em utilizar o jogo na sala de aula, o motivo apontado por ela foi a internet. Já as maiores dificuldades dos seus alunos segundo a mesma foi a internet e que os estudantes necessitavam de muita intervenção nas aulas para revisar o conteúdo antes das fases do jogo e de como jogá-lo, o que vai de encontro ao descrito na literatura por Fraser (2015), que afirma ser um tipo de jogo autodidata. Questionada em relação aos pontos positivos do jogo, ela comenta que os estudantes se sentiram bastante valorizados quando conseguiam passar de nível com a ajuda coletiva da turma para executar os comandos de programação em blocos e estudo dos ângulos.

\section{Conclusões}

O jogo Preservando a Natureza se mostrou adequado para o ensino de matemática, educação ambiental e lógica para discentes da turma de Jovens e Adultos, na amostra selecionada. Os estudantes demonstraram necessitar de muita intervenção nas aulas para revisar o conteúdo antes do início das fases do jogo e de como jogá-lo, o que vai de encontro ao descrito na literatura por Fraser (2015), o que traz indícios que a utilização desse tipo de jogo de forma autodidata necessita de estudos detalhados, em particular em relação ao público de jovens, adultos e idosos. O jogo promoveu a satisfação dos estudantes e todos os discentes que participaram do estudo gostariam de repetir a experiência com jogos em sala de aula.

\section{Referências}

FRASER, N. Ten Things We've Learned from Blockly. IEEE Blocks and Beyond Workshop, 2015.

FREITAS, F.A. DA, MAIA, P.H.M., BASÍLIO, J.O. PROGAME - Ambiente educacional gamificado com elementos dos descritores de matemática do sistema permanente de avaliação da educação básica do Ceará. VII Congresso Brasileiro de Informática na Educação (CBIE), 2018.GLUSHKOVAL, T. Application of Block programming and Game-Based Learning to Enhance Interest in Computer Science. Journal of innovations and Sustainability. n.1, p. 21-32, 2016.

GONZÁLES, L; GOMEZ,M.C.; ECHEVERRI, J. A. Motivation and Virtual Education in computer science: case universidad de Medellín-Colombia. 1176 IEEE Látin America Transactions, v. 15, n. 6, junho 2017.GOOGLE, Blockly Games. Disponível em: $<$ https://blockly-games.appspot.com/about?lang=en $>$ Acesso em 02 de janeiro de 2019.

HEIN, E., MASCHIO, E., MARCZAL, D., FILHO, P.L. RASPIBLOCOS: Ambiente de Programação Didático Baseado em Raspberry Pi e Blockly. Simpósio Brasileiro de Informática na Educação (SBIE), 2015.

LEITÃO, D.A., CASTRO, J.B.DE.A Construção de Recursos Digitais de Matemática: uma experiência de autoria com o Scratch. VII Congresso Brasileiro de Informática na Educação (CBIE), 2018.

MATOS, J.S.C., MATOS, F.B. O uso da Gamificação no projeto "Português Divertido", uma proposta de interdisciplinaridade. II Congresso sobre Tecnologias na Educação (Ctrl+E), 2017.

MEC, Trabalhando com a Educação de Jovens e Adultos :Alunos e Alunas do EJA. 2006. 
OLIVEIRA, T.M de, BITENCOURT, R., MONTEIRO, W.M. Experiências com Gamificação no Ensino de Computação para Jovens e Adultos no Sertão Pernambucano. Proceedings of SBGames 2016, 2016.

PAGANI, E.R, BOLZAN., M. de S. Ensinando Física através da Gamificação.VII Encontro Científico de Física Aplicada, 2016.

SILVA, J.B.DA, SALES, G.L., CASTRO, J.B. Gamificação de uma sequência didática como estratégia para motivar a atitude potencialmente significativa dos alunos no ensino de óptica geométrica. VII Congresso Brasileiro de Informática na Educação (CBIE), 2018.

SOUZA JUNIOR, J. C. de ; FIGUEIREDO, M. P.S. de ; MONTEIRO, Y.; CYSNEIROS, G. A. de A. .Realidade Virtual, Gamificação e Interdisciplinaridade, os norteadores para inovar a aula de História e de Geografia-Uma experiência vivenciada no Colégio CBV. V Congresso Nacional de Educação - CONEDU, 2018.

WILDSON, M.C., FRANZEN, E., GOMES, E.R. HELPBLOCK: uma ferramenta web baseada na biblioteca Blockly para apoio ao ensino de algoritmos. Revista Tecnologias na Educação , v. 25, 2018 